\title{
Higher Order Perturbation Theory for Exponential Lagrangians: Third Order*
}

\author{
K. POHLMEYeR** \\ Institute for Advanced Study, Princeton, New Jersey
}

Received November 14, 1971

Abstract. We define the vacuum expectation value of the time-ordered product of three exponentials of free massless fields as a continuous linear functional over a suitable test function space using minimal singularity as a criterion.

\section{Introduction}

The present paper is an extension of an earlier work [1] devoted to the analysis of the structure of exponential interactions as given by the Lagrangian $\mathscr{L}_{\text {int }}(f \phi)$

$$
\mathscr{L}_{\text {int }}(f \phi(x))=: e^{f \phi(x)}-1:=L_{\text {int }}(x)
$$

where $\phi$ is a free scalar field of mass $m$.

In [1] we discussed the second order contribution to the Green's functions in an expansion in powers of $\mathscr{L}_{\text {int }}(f \phi)$. To achieve uniqueness we introduced a minimality principle. We argued that with the least singular choice of the time-ordered product $T L_{\text {int }}\left(x_{1}\right) L_{\text {int }}\left(x_{2}\right)$ the Green's functions correspond most closely to the given classical Lagrangian (in second order).

Here we go one step beyond the results of Ref. [1] and show that the minimality principle can be generalized to third order, at least for the case of a massless field. The generalized minimality principle leads to a unique, least singular definition of the time-ordered product $T L_{\mathrm{int}}\left(x_{1}\right) \ldots L_{\mathrm{int}}\left(x_{3}\right)$. Because of the simple relation between time- and normal-ordered products of exponential Lagrangians it is sufficient to analyze the structure of the vacuum expectation values

$$
\begin{aligned}
\left\langle 0\left|T L_{\text {int }}\left(x_{1}\right) \ldots L_{\mathrm{int}}\left(x_{3}\right)\right| 0\right\rangle=\prod_{1 \leqq i<j \leqq 3}\left[e^{f^{2} i D_{F}\left(x_{1}-x_{j}\right)}-1\right] \\
\quad+\frac{1}{2} \sum_{\sigma \in \mathfrak{E}_{3}}\left[e^{f^{2} i D_{F}\left(x_{i}-x_{j}\right)}-1\right]\left[e^{f^{2} i D_{F}\left(x_{j}-x_{k}\right)}-1\right] \\
=\prod_{1 \leqq i<j \leqq 3} i E_{F}\left(x_{i}-x_{j}\right)+\frac{1}{2} \sum_{\sigma \in \mathfrak{S}_{3}}\left[i E_{F}\left(x_{i}-x_{j}\right)\right]\left[i E_{F}\left(x_{j}-x_{k}\right)\right] .
\end{aligned}
$$

$\star$ Research sponsored by the Alfred P. Sloan Foundation.

$\star \star$ On leave from II. Institut für Theoretische Physik der Universität Hamburg, Germany. 
Here $\mathfrak{S}_{3}$ denotes the group of permutations of three objects.

$$
\sigma(1,2,3)=(i, j, k) .
$$

The ambiguity in defining $\left\langle 0\left|T L_{\text {int }}\left(x_{1}\right) \ldots L_{\text {int }}\left(x_{3}\right)\right| 0\right\rangle$ consists in a translation and Lorentz invariant real symmetric distribution $\in \mathscr{C}^{\prime}\left(R^{12}\right)$ [2] the support of which is confined to $x_{1}=x_{2}=x_{3}$. Hence the second term on the r.h.s. of (2) although already defined may be replaced by

$$
\begin{gathered}
\frac{1}{2} \sum_{\sigma \in \mathfrak{\subseteq}_{3}}\left\{\left[i E_{F}\left(x_{i}-x_{j}\right)\right]\left[i E_{F}\left(x_{j}-x_{k}\right)\right]-\pi^{6} \lambda^{4}\left[g\left(-\frac{\lambda}{4} \square\right) \delta\left(x_{i}-x_{j}\right)\right]\right. \\
\left.\cdot\left[g\left(-\frac{\lambda}{4} \square\right) \delta\left(x_{j}-x_{k}\right)\right]\right\}
\end{gathered}
$$

with the least singular superpropagator $i E_{F}(x), \lambda=f^{2} / 4 \pi^{2}$ and

$$
g(Z)=\sum_{m=0}^{\infty} \frac{Z^{m}}{m !(m+1) !(m+2) !} .
$$

The real part of the Fourier transform of expression (4) is given by

$$
\begin{aligned}
& \frac{-\pi^{2} \lambda^{4}}{8} \delta\left(\sum_{1}^{3} p_{n}\right) \sum_{\sigma \in \mathfrak{G}_{3}}\left\{\operatorname{Re} \tilde{E}_{F}\left(p_{i}\right) \cdot \operatorname{Re} \tilde{E}_{F}\left(p_{k}\right)-\pi^{2}\left[\Theta\left(-p_{i}^{2}\right) g\left(\frac{\lambda}{4} p_{i}^{2}\right)-\delta\left(p_{i}^{2}\right)\right]\right. \\
& \cdot\left[\Theta\left(-p_{k}^{2}\right) g\left(\frac{\lambda}{4} p_{k}^{2}\right)-\delta\left(p_{k}^{2}\right)\right]+\pi^{2}\left(\left[\Theta\left(-p_{i}^{2}\right) g\left(\frac{\lambda}{4} p_{i}^{2}\right)-\delta\left(p_{i}^{2}\right)\right] \cdot g\left(\frac{\lambda}{4} p_{k}^{2}\right)\right. \\
& +[i \leftrightarrow k])\} .
\end{aligned}
$$

Suppose that (6) is smeared in the spatial variables with a testfunction $\tilde{\phi}\left(\boldsymbol{p}_{1}, \boldsymbol{p}_{2}, \boldsymbol{p}_{3}\right) \in \mathscr{D}\left(R^{9}\right)[3]$ such that there exists a constant $K$ with

Hence

$$
0 \leqq \boldsymbol{p}_{n}^{2}<K^{2} \quad n=1,2,3 \text { for }\left(\boldsymbol{p}_{1}, \cdot, \boldsymbol{p}_{3}\right) \in \operatorname{supp} \tilde{\phi} .
$$

$$
p_{n, 0}^{2}-K^{2}<p_{n}^{2} \quad n=1,2,3 .
$$

The various terms of (6) smeared with $\tilde{\phi}$ are of the following type for large $\left|p_{i, 0}\right|$ or $\left|p_{k, 0}\right|$ :

$$
\begin{gathered}
\delta\left(\sum p_{n, 0}\right)\left\{O\left(\operatorname{Max}^{-N}\left\{\left|p_{i, 0}\right|,\left|p_{k, 0}\right|\right\}\right)+\left(O\left(\left|p_{i, 0}\right|^{-N}\right) \times\right. \text { entire function }\right. \\
\left.\left.\quad \text { of order } 2 / 3 \text { in } p_{k, 0}+[i \leftrightarrow k]\right)\right\}
\end{gathered}
$$

for any $N \in \mathbb{N}$. 

for

With the help of this information one obtains the following structure

$$
\begin{gathered}
\frac{1}{2} \sum_{\sigma \in \mathbb{S}_{3}}\left\langle\operatorname { R e } \left\{\left[i E_{F}\left(x_{i}-x_{j}\right)\right]\left[i E_{F}\left(x_{j}-x_{k}\right)\right]-\pi^{6} \lambda^{4}\left[g\left(-\frac{\lambda}{4} \square\right) \delta\left(x_{i}-x_{j}\right)\right]\right.\right. \\
\left.\left.\cdot\left[g\left(-\frac{\lambda}{4} \square\right) \delta\left(x_{j}-x_{k}\right)\right]\right\}, \phi\left(\boldsymbol{x}_{1}, \boldsymbol{x}_{2}, \boldsymbol{x}_{3}\right)\right\rangle
\end{gathered}
$$

as a function of the time differences

with

$$
\sum_{\sigma \in \mathfrak{S}_{3}}\left\{h^{\sigma}\left(x_{i}^{0}-x_{j}^{0}, x_{j}^{0}-x_{k}^{0}\right)+\sum_{v=0}^{\infty} \delta^{(v)}\left(x_{i}^{0}-x_{j}^{0}\right) h_{v}^{\sigma}\left(x_{j}^{0}-x_{k}^{0}\right)\right\}
$$

and

$$
h^{\sigma} \in C^{\infty}\left(R^{2}\right), \quad h_{v}^{\sigma} \in C^{\infty}\left(R^{1}\right) \quad v=0,1,2, \ldots
$$

$$
\sum_{v=0}^{\infty} \delta^{(v)}\left(x_{i}^{0}-x_{j}^{0}\right) h_{v}^{\sigma}\left(x_{j}^{0}-x_{k}^{0}\right) \in \mathscr{C}^{\prime}\left(R^{2}\right) \text { for all } \sigma \in \mathbb{S}_{3} .
$$

The singularities are contained in the second term of (11). They are attached to the lines $x_{1}^{0}=x_{2}^{0}, x_{2}^{0}=x_{3}^{0}$ and $x_{3}^{0}=x_{1}^{0}$. In particular, for no spatial testfunction $\phi$ does the expression (10) involve singularities belonging to the point $x_{1}=x_{2}=x_{3}$.

The crux of the problem consists of showing that in the weighted space average of the real part of the first term on the r.h.s. of (2) background, line singularities and point singularity can be separated from each other. Once this has been proved one particular definition of $\prod_{1 \leqq i<j \leqq 3}\left[i E_{F}\left(x_{i}-x_{j}\right)\right]$ can be singled out by the absence of point singularities in the weighted space averages of its real part or in other words by its being least singular. Then the vacuum expectation value of the time ordered product $T L_{\text {int }}\left(x_{1}\right) \ldots L_{\text {int }}\left(x_{3}\right)$ is defined by the sum of that particular definition of

$$
\begin{gathered}
\prod_{1 \leq i<j \leq 3}\left[i E_{F}\left(x_{i}-x_{j}\right)\right] \text { plus } \frac{1}{2} \sum_{\sigma \in \Theta_{3}}\left\{\left[i E_{F}\left(x_{i}-x_{j}\right)\right]\left[i E_{F}\left(x_{j}-x_{k}\right)\right]\right. \\
\left.-\pi^{6} \lambda^{4}\left[g\left(-\frac{\lambda}{4} \square\right) \delta\left(x_{i}-x_{j}\right)\right]\left[g\left(-\frac{\lambda}{4} \square\right) \delta\left(x_{j}-x_{k}\right)\right]\right\} .
\end{gathered}
$$

Clearly, the weighted space averages of the real part of $\langle 0| T L_{\text {int }}\left(x_{1}\right) \ldots$ $\ldots L_{\text {int }}\left(x_{3}\right)|0\rangle$ again do not contain point singularities. Hence $\left\langle 0\left|T L_{\mathrm{int}}\left(x_{1}\right) \ldots L_{\mathrm{int}}\left(x_{3}\right)\right| 0\right\rangle$ so defined is least singular.

In Section II we give a particular definition for $\prod_{1 \leqq i<j \leqq 3}\left[i E_{F}\left(x_{i}-x_{j}\right)\right]$ and prove that it satisfies unitarity and locality. In Section III we show that this particular definition is already suggested by the behavior of $\prod_{1 \leqq i<j \leqq 3}\left[i E_{F}\left(x_{i}-x_{j}\right)\right]$ in the neighborhood of the point $x_{1}=x_{2}=x_{3}$ and 
consequently is the least singular one. We conclude with a remark concerning the singularity structure in the invariant momenta of the corresponding amplitude in momentum space.

It will not escape the reader's attention that all of the preceding and subsequent considerations carry easily over to Lagrangians of the form

$$
\mathscr{L}_{\text {int }}(f \phi)=\int_{0}^{\infty} d \mu(q): e^{q f \phi}-1:
$$

where $\left[\exp q^{\alpha}\right] d \mu(q)$ is a bounded real measure for some $\alpha>2$.

\section{Definition of the Time-Ordered Product, Unitarity and Locality}

To prepare the ground for the subsequent discussion, let us investigate powers

and

$$
\left[Q\left(p_{1}, p_{2} ; t_{1}, t_{2}\right) \pm i 0\right]^{\mu}
$$

$$
\left[Q\left(p_{1}, p_{2} ; t_{1}, t_{2}\right) \pm i \varepsilon\right]^{\mu}
$$

of the parameter depending quadratic form

$$
Q\left(p_{1}, p_{2} ; t_{1}, t_{2}\right)=-\frac{p_{1}^{2}+t_{1} p_{2}^{2}+t_{1} t_{2}\left(p_{1}+p_{2}\right)^{2}}{1+t_{2}+t_{2} t_{1}}
$$

for $\operatorname{Re} \mu>-4, t_{j} \in I=[0,1] j=1,2$ and $\varepsilon>0[3]$.

As a distribution-valued function $\left[Q\left(p_{1}, p_{2} ; t_{1}, t_{2}\right) \pm i 0\right]^{\mu}$ is infinitely differentiable with respect to $t_{1}$ and $t_{2}$ as long as the quadratic form $Q\left(p_{1}, p_{2} ; t_{1}, t_{2}\right)$ is not degenerate, i.e. away from $t_{1}=0$. For $\operatorname{Re} \mu>-4$ the behavior of (13) when $t_{1}$ approaches the left end of $I$ is given by

$$
\left[Q\left(p_{1}, p_{2} ; t_{1}, t_{2}\right) \pm i 0\right]^{\mu}=G_{\mu}\left(p_{1}, p_{2} ; t_{1}, t_{2}\right)+t_{1}^{\mu+2} G_{\mu}^{\prime}\left(p_{1}, p_{2} ; t_{1}, t_{2}\right)
$$

where the distribution-valued function $G_{\mu}$ is infinitely differentiable with respect to $t_{1}$ and $t_{2}$ and where $G_{\mu}^{\prime}$ is a bounded function of $t_{1}$ and $t_{2}$ for $t_{j} \in I, j=1,2$ (bounded in the sense of $\left.\mathscr{S}^{\prime}\right)^{1}$. Moreover, we have

$$
\begin{gathered}
\lim _{\varepsilon \downarrow 0}\left[Q\left(p_{1}, p_{2} ; t_{1}, t_{2}\right) \pm i \varepsilon\right]^{\mu} \\
=\left[Q\left(p_{1}, p_{2} ; t_{1}, t_{2}\right) \pm i 0\right]^{\mu} \text { for } \operatorname{Re} \mu>-4 \text { and } t_{1} \neq 0 .
\end{gathered}
$$

${ }^{1}$ Qualitatively, this behavior can be seen from the partial Fourier transform with respect to the variable $p_{1}$. It is a special case of Lemma 2.2.20 in E. R. Speer and M. J. Westwater, Generic Feyman Amplitudes, IAS preprint (1970). However, also in the general situation, an alternative, more direct proof of the corresponding behavior when the parameter depending quadratic form $Q$ degenerates can be given which does not involve analytic continuation from the Symanzik region. 
Thus the distribution

$$
\begin{aligned}
\delta\left(\sum_{l=1}^{3} p_{l}\right) T_{0}^{\sigma \pm}\left(\{p\} ; s_{1}, s_{2}, s_{3}\right) & \\
= & \delta\left(\sum_{l=1}^{3} p_{l}\right) \int_{0}^{1} d t_{2} t_{2}^{s_{3}+1} \int_{0}^{1} d t_{1} t_{1}^{-s_{1}-1}\left[1+t_{2}+t_{2} t_{1}\right]^{-2} \\
& \cdot\left[Q\left(p_{i}, p_{j} ; t_{1}, t_{2}\right) \pm i 0\right]^{s_{3}+s_{2}+s_{1}+2},
\end{aligned}
$$

unambiguously defined in

$$
\left\{s_{1}, s_{2}, s_{3} / \operatorname{Re} s_{1}<0, \operatorname{Re} s_{3}>-2, \sum_{i=1}^{3} \operatorname{Re} s_{i}>-2\right\},
$$

can be analytically continued in $s_{1}, s_{2}, s_{3}$ to a function meromorphic in $\Omega$

$$
\Omega=\left\{s_{1}, s_{2}, s_{3} / \sum_{k=l+1}^{3} \operatorname{Re} s_{k}>-2(3-l) \text { for all } 0 \leqq l \leqq 2\right\} .
$$

If we use the same symbol for the continued function, then

$$
\delta\left(\sum_{l=1}^{3} p_{l}\right) \Gamma\left(-s_{1}\right)^{-1} T_{0}^{\sigma \pm}\left(\{p\} ; s_{1}, s_{2}, s_{3}\right)
$$

is analytic in $\Omega$. We define

$$
\begin{aligned}
\delta\left(\sum_{l=1}^{3} p_{l}\right) T_{\varepsilon}^{\sigma \pm}\left(\{p\} ; s_{1}, s_{2}, s_{3}\right) \\
=\delta\left(\sum_{l=1}^{3} p_{l}\right) \int_{0}^{1} d t_{2} t_{2}^{s_{3}+1} \int_{0}^{1} d t_{1} t_{1}^{-s_{1}-1}\left[1+t_{2}+t_{2} t_{1}\right]^{-2} \\
\cdot\left[Q\left(p_{i}, p_{j} ; t_{1}, t_{2}\right) \pm i \varepsilon\right]^{s_{3}+s_{2}+s_{1}+2} .
\end{aligned}
$$

Then for $\{s\} \in \Omega$, there exists the limit in the sense of $\mathscr{S}^{\prime}\left(R^{12}\right)$

$$
\lim _{\varepsilon \downarrow 0} \delta\left(\sum_{l=1}^{3} p_{l}\right) \Gamma\left(-s_{1}\right)^{-1} T_{\varepsilon}^{\sigma \pm}\left(\{p\} ; s_{1}, s_{2}, s_{3}\right)
$$

and is equal to the corresponding distribution (19).

For the moments $p_{1}, p_{2}, p_{3}$ in a compact set $K$, i.e. in $\mathscr{D}^{\prime}(K)$ the distributions (20) are equal to

$$
\begin{gathered}
-\delta\left(\sum_{l=1}^{3} p_{l}\right) \frac{\pi}{\sin \pi s_{1}} \int_{0}^{1} d t_{2} t_{2}^{s_{3}+1} \frac{1}{2 \pi i} \int_{C_{K, \varepsilon}} d t_{1}\left(-t_{1}\right)^{-s_{1}-1}\left[1+t_{2}+t_{2} t_{1}\right]^{-2} \\
\cdot\left[Q\left(p_{i}, p_{j} ; t_{1}, t_{2}\right) \pm i \varepsilon\right]^{\sum s_{l}+2} .
\end{gathered}
$$


Here the contour $C_{K, \varepsilon}$ depending on $K$ and $\varepsilon$ starts and ends at +1 and encircles the origin once counter-clockwise. It is so close to the real interval $[0,1]$ that $Q\left(p_{i}, p_{j} ; t_{1}, t_{2}\right) \pm i \varepsilon$ does not vanish in

$$
\left\{t_{1} / t_{1} \in \text { region encircled by } C_{K, \varepsilon}\right\} \times\left\{t_{2} / t_{2} \in I\right\} \text {. }
$$

The function $(-t)^{-s_{1}-1}$ is defined such that $\arg (-t)=0$ for $t<0$.

Next, we turn to the asymptotic behavior of the distributions

$$
\delta\left(\sum_{l=1}^{3} p_{l}\right) \Gamma\left(-s_{1}\right)^{-1} T_{\varepsilon}^{\sigma \pm}\left(\{p\} ; s_{1}, s_{2}, s_{3}\right) \text { for } \varepsilon \geqq 0 .
$$

We expand

$$
\begin{gathered}
{\left[-\left(1+t_{2}\right)^{-1} \cdot p_{1}^{2}-\tau\left(1+t_{2}\right)\left(1+t_{2}+t_{2} t_{1}\right)^{-1}\right.} \\
\left.\cdot\left(\frac{t_{2}}{1+t_{2}} p_{1}+p_{2}\right)^{2} \pm i \varepsilon\right]^{\sum_{1}^{3} s_{l}+2} \text { for } \varepsilon \geqq 0
\end{gathered}
$$

with respect to $\tau$ using the formula

$$
f(x)=\sum_{n=0}^{N} \frac{x^{n}}{n !} f^{(n)}(0)+\frac{1}{N !} \int_{0}^{1} d v(1-v)^{N} \frac{\partial^{N+1}}{\partial v^{N+1}} f(v x) \text { for } f \in C^{N+1}\left(R^{1}\right),
$$

set $\tau$ equal to $t_{1}$ and choose $N$ such that $3+\sum_{1}^{3} \operatorname{Re} s_{l} \leqq N<4+\sum_{1}^{3} \operatorname{Re} s_{l}$. This expansion leads to a corresponding expansion for (24) a term by term estimate of which gives the following result:

There exists a positive constant $M$ such that the set of distributions

$$
\begin{aligned}
& \left\{\left(M\left[1+\sum_{l=1}^{3} \sum_{v=0}^{3}\left|p_{l, v}\right|^{2}\right]\left[1+\frac{\left[\sum_{1}^{3} \operatorname{Im} s_{l}\right]^{2}}{\left[\operatorname{Max}\left\{1, \sum_{1}^{3} \operatorname{Re} s_{l}+2\right\}\right]^{2}}\right]^{\frac{1}{2}}\right]^{-\sum_{1}^{3} \operatorname{Re} s_{l}-6}\right. \\
& \cdot e^{-\pi\left|\operatorname{Im} s_{1}\right|}\left|\Gamma\left(1+s_{1}\right)\right|^{-1} e^{-\pi\left|\sum_{1}^{3} \operatorname{Im} s_{l}\right|} \delta\left(\sum_{1}^{3} p_{l}\right) \Gamma\left(-s_{1}\right)^{-1} T_{\varepsilon}^{\sigma \pm}\left(\{p\} ; s_{1}, s_{2}, s_{3}\right) \\
& \left./\left(s_{1}, s_{2}, s_{3}\right) \in \Omega, 0 \leqq \varepsilon \leqq 1\right\}=B \subset \mathscr{S}^{\prime}\left(R^{12}\right)
\end{aligned}
$$

is bounded. 
We are now in a position to define

and

$$
\mathscr{T}\left(x_{1}, x_{2}, x_{3}\right)=\prod_{1 \leqq j<k \leqq 3}\left[i E_{F}\left(x_{j}-x_{k}\right)\right]
$$

$$
\overline{\mathscr{T}}\left(x_{1}, x_{2}, x_{3}\right)=\prod_{1 \leqq j<k \leqq 3}\left[-i E_{\bar{F}}\left(x_{j}-x_{k}\right)\right]
$$

in momentum space where

$$
\tilde{E}_{F(\bar{F})}(k)=\frac{\lambda^{2}}{4} \frac{1}{2 \pi i} \int_{L} d s \frac{\Gamma(-1-s) \Gamma(-s) \cos \pi s}{\Gamma(3+s)}\left[\frac{\lambda}{4}\left(-k_{(\mp)}^{2} i 0\right)\right]^{s} .
$$

The loop $L$ starts and ends at $+\infty$ and encircles the poles $-1,0,+1, \ldots$ once clockwise. $\lambda=\frac{f^{2}}{4 \pi^{2}}$ is assumed to be positive.

$$
\left[\tilde{E}_{F}(k)\right]^{*}=\tilde{E}_{\bar{F}}(k), \text { i.e. }\left[E_{F}(x)\right]^{*}=E_{\bar{F}}(x) .
$$

\section{Definition.}

$$
\begin{aligned}
& \mathscr{F}_{x_{1}, x_{2}, x_{3}}\left\{\mathscr{T}\left(x_{1}, x_{2}, x_{3}\right)\right\}\left(p_{1}, p_{2}, p_{3}\right)=\tilde{\mathscr{T}}\left(p_{1}, p_{2}, p_{3}\right) \\
&=\frac{\pi^{2} \lambda^{4}}{4} \sum_{0}^{\infty} \frac{1}{m_{3} !\left(m_{3}+1\right) !} \sum_{0}^{\infty} \frac{1}{m_{2} !\left(m_{2}+1\right) !} \\
& \cdot \frac{1}{2 \pi i} \int_{C} d s_{1} \frac{\Gamma\left(-1-s_{1}\right)}{\Gamma\left(3+s_{1}\right)} \cos \pi s_{1}\left[1+\sin ^{2} \pi s_{1}\right] \\
& \cdot \Gamma\left(-m_{3}-m_{2}-s_{1}\right)\left(\frac{\lambda}{4}\right)^{m_{3}+m_{2}+s_{1}} \\
& \cdot \sum_{\sigma \in \mathfrak{\Xi}_{3}} \delta\left(\sum_{1}^{3} p_{n}\right) T_{0}^{\sigma-}\left(\{p\} ; s_{1}, m_{2}-1, m_{3}-1\right)+\tilde{\mathfrak{R}}^{-}
\end{aligned}
$$

where the loop $C$ starts and ends at $+\infty$ and encircles the points $-2,-1$, $0,+1, \ldots$ once clockwise and where $\tilde{\mathfrak{R}}^{-}$is given by

$$
\begin{aligned}
& \tilde{\mathfrak{R}}^{-}=\frac{\pi^{2} \lambda^{4}}{4} \sum_{0}^{\infty}\left(\frac{\lambda}{4}\right)^{l} \frac{1}{l !} \frac{1}{(2 \pi i)^{2}} \int_{S_{2}-i \infty}^{S_{2}+i \infty} d s_{2} \int_{S_{1}-i \infty}^{s_{1}+i \infty} d s_{1} \prod_{n=1}^{2} \\
& \cdot\left[\frac{\Gamma\left(-1-s_{n}\right)}{\Gamma\left(3+s_{n}\right)}\right] \frac{\Gamma\left(s_{2}+s_{1}+1-l\right)}{\Gamma\left(l+1-s_{1}-s_{2}\right)} \sum_{\sigma \in \Theta_{3}} \delta\left(\sum_{1}^{3} p_{n}\right) T_{0}^{\sigma-}\left(\{p\} ; s_{1}, s_{2}, l-2-s_{1}-s_{2}\right) \\
& S_{2}<-1, S_{1}<-2,-7 / 2<S_{2}+S_{1}<-3 .
\end{aligned}
$$$$
\tilde{\tilde{\mathscr{T}}}\left(p_{1}, p_{2}, p_{3}\right) \text { is obtained from } \tilde{\mathscr{T}}\left(p_{1}, p_{2}, p_{3}\right) \text { by replacing } T_{0}^{\sigma-} \text { by } T_{0}^{\sigma+} \text {. }
$$ 
We note the relation

$\left[\tilde{\mathscr{T}}\left(p_{1}, p_{2}, p_{3}\right)\right]^{*}=\tilde{\mathscr{\mathscr { T }}}\left(p_{1}, p_{2}, p_{3}\right)$, i.e. $\left[\mathscr{T}\left(x_{1}, x_{2}, x_{3}\right)\right]^{*}=\tilde{\mathscr{T}}\left(x_{1}, x_{2}, x_{3}\right)$.

Using (27) and standard estimates on gamma and beta functions one verifies that

$$
\tilde{\mathscr{T}}\left(p_{1}, p_{2}, p_{3}\right) \in \mathfrak{M}_{1 / 3}^{\prime}\left(R^{12}\right) \text { and } \quad \tilde{\mathscr{T}}\left(p_{1}, p_{2}, p_{3}\right) \in \mathfrak{M}_{1 / 3}^{\prime}\left(R^{12}\right)
$$

where $\mathfrak{M}_{1 / 3}^{\prime}\left(R^{l}\right)$ denotes the space of all linear continuous functionals over the test function space

$$
\begin{gathered}
\mathfrak{M}_{1 / 3}\left(R^{l}\right)=\left\{\tilde{f}\left(q_{1}, \ldots, q_{l}\right) / \tilde{f} \in C^{\infty}\left(R^{l}\right),\langle\tilde{f}\rangle_{d}^{(\alpha)}=\sup _{q} \mid g\left(d\|q\|^{2}\right)\right. \\
\left.D^{(\alpha)} \tilde{f}\left(q_{1}, \ldots, q_{l}\right) \mid<\infty \text { for all } d=1,2, \ldots \text { and all }(\alpha) \in \mathbb{N}^{l}\right\} \\
D^{(\alpha)}=\prod_{j=1}^{l}\left(\frac{\partial}{\partial q_{j}}\right)^{\alpha j}, \quad\|q\|^{2}=\sum_{j=1}^{l} q_{j}^{2} .
\end{gathered}
$$

$\mathfrak{M}_{1 / 3}\left(R^{l}\right)$ is equipped with the topology given by the norms $\langle\tilde{f}\rangle_{d}^{(\alpha)}[2]$.

In order to prove that the above definitions of $\mathscr{T}$ and $\mathscr{T}$ are permissible in the sense that they satisfy unitarity and locality, we consider the following functions of an auxiliary parameter $\gamma$, first for $\gamma$ real and larger than nine ${ }^{2}$

$$
\begin{aligned}
& \stackrel{(\tilde{T})}{T}_{r}\left(p_{1}, p_{2}, p_{3} ; \gamma\right)=\frac{\pi^{2} \lambda^{4}}{4} \frac{1}{(2 \pi i)^{3}} \int_{S-i \infty}^{S+i \infty} \int_{-i \infty} d s_{3} d s_{2} d s_{1} \prod_{j=1}^{3}\left[\frac{\Gamma\left(-\gamma\left(1+s_{j}\right)\right)}{\Gamma\left(3+s_{j}\right)}\right] \\
& \cdot\left[\frac{\lambda}{4} e^{i \pi r}\right]^{\sum_{1}^{3} s_{j}+2} \Gamma\left(-\sum_{1}^{3} s_{j}-2\right) \sum_{\sigma \in \mathbb{E}_{3}} \delta\left(\sum_{1}^{3} p_{n}\right) T_{0}^{\sigma(\mp)}\left(\{p\} ; s_{1}, s_{2}, s_{3}\right), \\
& r= \pm 1, \pm 3 ;-\frac{3}{2}<S<-1 \text {. }
\end{aligned}
$$

With the help of (27) and standard estimates the integrals can be shown to exist for these values of $\gamma$. However, for values of $\gamma$ close to +1 they will not exist as they stand. We swing the $s_{3}$ - and $s_{2}$-contours around the real axis from -1 to $+\infty$ which will push the $s_{1}$-contour to the far left. Subdividing this deformed $s_{1}$-contour into 2 parts: one parallel to the imaginary axis: $\operatorname{Re} s_{1}=S$, the other one being a loop which encircles the poles of $\Gamma\left(-\sum_{1}^{3} s_{j}-2\right)$ with $\operatorname{Re} s_{1} \leqq S$ once clockwise (a singularity on $\operatorname{Re} s_{1}=S$ is to be accounted for by either the first or the second part, but

2 This type of regularization was first introduced by M. K. Volkov in Ann. Phys. (N.Y.) 49, 202 (1968). 
not by both) we derive the following identity

$$
\begin{aligned}
\stackrel{(\widetilde{T}}{r}_{r}\left(p_{1}, p_{2}, p_{3} ; \gamma\right)=\frac{\pi^{2} \lambda^{4}}{4} \frac{1}{\gamma^{2}} \sum_{0}^{\infty} \frac{(-1)^{m_{3}}}{m_{3} ! \Gamma\left(2+m_{3} / \gamma\right)} \sum_{0}^{\infty} \frac{(-1)^{m_{2}}}{m_{2} ! \Gamma\left(2+m_{2} / \gamma\right)} \\
\cdot \frac{1}{2 \pi i} \int_{S-i \infty}^{s+i \infty} d s_{1} \frac{\Gamma\left(-\gamma\left(1+s_{1}\right)\right)}{\Gamma\left(3+s_{1}\right)}\left[\frac{\lambda}{4} e^{i \pi r}\right]^{\left(m_{3} / \gamma\right)+\left(m_{2} / \gamma\right)+s_{1}} \\
\cdot \Gamma\left(-\frac{m_{3}}{\gamma}-\frac{m_{2}}{\gamma}-s_{1}\right) \Gamma\left(-s_{1}\right) \sum_{\sigma \in \mathfrak{S}_{3}} \delta\left(\sum_{1}^{3} p_{n}\right) \Gamma\left(-s_{1}\right)^{-1} \\
\cdot T_{0}^{\sigma(\overline{+})}\left(\{p\} ; s_{1}, \frac{m_{2}}{\gamma}-1, \frac{m_{3}}{\gamma}-1\right)+\tilde{\mathfrak{R}}^{(\mp)}(\gamma)
\end{aligned}
$$

where

$$
\begin{aligned}
\check{\mathfrak{R}}^{(\mp)}(\gamma) & =\frac{\pi^{2} \lambda^{4}}{4} \sum_{0}^{\infty} \frac{\left(\frac{\lambda}{4}\right)^{l}}{l !}\left\{\frac{1}{(2 \pi i)^{2}} \int_{S-i \infty}^{s+i \infty} d s_{2} d s_{1} \prod_{j=1}^{2} \frac{\Gamma\left(-\gamma\left(1+s_{j}\right)\right)}{\Gamma\left(3+s_{j}\right)}\right. \\
& \cdot \frac{\Gamma\left(\gamma\left[s_{2}+s_{1}+1-l\right]\right)}{\Gamma\left(l+1-s_{1}-s_{2}\right)} \sum_{\sigma \in \mathfrak{S}_{3}} \delta\left(\sum_{1}^{3} p_{n}\right) T_{0}^{\sigma(\mp)}\left(\{p\} ; s_{1}, s_{2}, l-2-s_{1}-s_{2}\right) \\
+ & \frac{1}{2 \pi i} \int_{Z} d s_{3} \frac{\Gamma\left(-\gamma\left(1+s_{3}\right)\right) \cos \pi \gamma\left(1+s_{3}\right)}{\Gamma\left(3+s_{3}\right)} \frac{1}{2 \pi i} \int_{S-i \infty}^{S+i \infty} d s_{1} \frac{\Gamma\left(-\gamma\left(1+s_{1}\right)\right)}{\Gamma\left(3+s_{1}\right)} \\
& \cdot \frac{\pi}{\sin \pi \gamma\left(s_{1}-l\right)} \cdot \Gamma\left(1+\gamma\left[l-1-s_{1}-s_{3}\right]\right)^{-1} \Gamma\left(1+l-s_{1}-s_{3}\right)^{-1} \\
& \left.\cdot \sum_{\sigma \in \mathfrak{\Xi}_{3}} \delta\left(\sum_{1}^{3} p_{n}\right) T_{0}^{\sigma(\mp)}\left(\{p\} ; s_{1}, l-2-s_{1}-s_{3}, s_{3}\right)\right\} .
\end{aligned}
$$

$S$ is the same in both formulae (37) and (38) and $-\frac{3}{2}<S<-1$. The contour $Z$ encircles those of the poles $s_{3}=-1+\frac{m_{3}}{\gamma}, m_{3}=0,1, \ldots$ once clockwise whose real part is less or equal to $l-2-2 S$.

In much the same way as before, one shows from this form of $\stackrel{(\mathscr{T}}{r}_{r}$ that

$$
{\stackrel{(\widetilde{\sigma})}{\mathscr{T}_{r}}}_{r}\left(p_{1}, p_{2}, p_{3} ; \gamma\right) \in \mathfrak{M}_{1 / 3}^{\prime}\left(R^{12}\right)
$$

and that $\tilde{\mathscr{T}}_{r}\left(p_{1}, p_{2}, p_{3} ; \gamma\right)$ and $\tilde{\widetilde{T}}_{r}\left(p_{1}, p_{2}, p_{3} ; \gamma\right)$ are analytic functions of $\gamma$ in the chisel shaped region $W_{\delta}$

$$
W_{\delta}=\left\{\gamma=\gamma_{1}+i \gamma_{2} / \gamma_{1}>1,\left|\gamma_{2}\right|<\operatorname{Min}\left[\delta, \frac{\pi}{4}\left(\gamma_{1}-1\right)\right]\right\}, \quad \delta>0 .
$$

Furthermore, the following limits exist in $\mathfrak{M}_{1 / 3}^{\prime}$ :

$$
\lim _{\substack{\gamma \rightarrow 1 \\ \gamma \in W_{\delta}}}\left\{\frac{5}{8} \sum_{r= \pm 1} \stackrel{(\tilde{\sim})}{\tilde{T}_{r}}\left(p_{1}, p_{2}, p_{3} ; \gamma\right)-\frac{1}{8} \sum_{r= \pm 3}{\stackrel{(\tilde{T})}{\mathscr{T}_{r}}}_{r}\left(p_{1}, p_{2}, p_{3} ; \gamma\right)\right\}
$$


and are equal to $\tilde{\mathscr{T}}\left(p_{1}, p_{2}, p_{3}\right)$ and $\tilde{\mathscr{T}}\left(p_{1}, p_{2}, p_{3}\right)$ respectively. Next, we introduce the parameter depending superpropagators

$$
\begin{gathered}
\tilde{E}_{F(\bar{F}), r}(k ; \gamma)=\frac{\lambda^{2}}{4} \frac{1}{2 \pi i} \int_{L_{\gamma}} d s \frac{\Gamma(-\gamma(1+s)) \Gamma(-s)}{\Gamma(3+s)} e^{i \pi r s}\left[\frac{\lambda}{4}\left(\boldsymbol{k}^{2}-k_{0(\mp)}^{2} i 0\right)\right]^{s} \\
r= \pm 1, \pm 3
\end{gathered}
$$

where the loop $L_{\gamma}$ encircles the poles of the gamma functions once clockwise. Using Stirling's formula and Jensen's inequality one shows:

a) $\tilde{E}_{F(\bar{F}), r}(k ; \gamma) \in \mathfrak{M}_{1 / 3}^{\prime}\left(R^{4}\right)$ for $\operatorname{Re} \gamma \geqq 1$,

b) $\tilde{E}_{F(\bar{F}), r}(k ; \gamma)$ are analytic functions of $\gamma$ in $W_{\delta}$,

c) the following limits exist in $\mathfrak{M}_{1 / 3}^{\prime}\left(R^{4}\right)$

and are equal to

$$
\lim _{\substack{\gamma \rightarrow+1 \\ \gamma \in W_{\delta}}} \tilde{E}_{F(\bar{F}), r}(k ; \gamma)
$$

$$
\tilde{E}_{F(\bar{F}), r}(k)=\frac{\lambda^{2}}{4} \frac{1}{2 \pi i} \int_{L} d s \frac{\Gamma(-1-s) \Gamma(-s)}{\Gamma(3+s)} e^{i \pi r s}\left[\frac{\lambda}{4}\left(-k_{(\overline{+})}^{2} i 0\right)\right]^{s} .
$$

For the difference $E_{F, r}$ and $E_{\bar{F}, r}$ one finds

$$
\begin{aligned}
\tilde{E}_{F, r}(k ; \gamma)-\tilde{E}_{\bar{F} . r}(k ; \gamma)= & -2 \pi i \frac{\lambda}{\gamma} \delta\left(k^{2}\right) \\
& -2 \pi i \frac{\lambda^{2}}{4 \gamma} \sum_{0}^{\infty} \frac{(-1)^{m}\left[\frac{\lambda}{4} e^{i \pi r}\right]^{(m+1) / \gamma-1}\left(\frac{m+1}{\gamma}\right) \Gamma(m+2) \Gamma\left(\frac{m+1}{\gamma}+2\right)}{\Gamma\left(\frac{m+1) / \gamma-1}{+}\right)} \\
= & \tilde{E}_{r}^{(+)}(k ; \gamma)+\tilde{E}_{r}^{(-)}(k ; \gamma)
\end{aligned}
$$

with

$$
\begin{aligned}
\tilde{E}_{r}^{( \pm)}(k ; \gamma)= & -2 \pi i \frac{\lambda}{\gamma} \delta_{ \pm}\left(k^{2}\right)+\frac{\lambda^{2}}{4} \Theta\left( \pm k_{0}\right) \int_{L_{\gamma}^{\prime}} d s \frac{\Gamma(-\gamma(1+s))}{\Gamma(1+s) \Gamma(3+s)} \\
& \cdot\left[\frac{\lambda}{4} e^{i \pi r}\right]^{s}\left(k^{2}\right)_{+}^{s}
\end{aligned}
$$

where the loop $L_{\gamma}^{\prime}$ encircles the poles $s=-1+\frac{m}{\gamma} m=1,2, \ldots$ once clockwise. $E^{(+)}$and $E^{(-)}$are related by

$$
E_{r}^{(+)}(x ; \gamma)=E_{r}^{(-)}(-x ; \gamma) .
$$


Going through the same routine as before, one shows that

a) $\tilde{E}_{r}^{( \pm)}(k ; \gamma) \in \mathfrak{M}_{1 / 3}^{\prime}\left(R^{4}\right)$ for $\operatorname{Re} \gamma \geqq 1$.

b) $\tilde{E}_{r}^{( \pm)}(k ; \gamma)$ are analytic functions of $\gamma$ in $W_{\delta}$.

c) the following limits exist in $\mathfrak{M}_{1 / 3}^{\prime}\left(R^{4}\right)$

$$
\lim _{\substack{\gamma \rightarrow+1 \\ \gamma \in W_{\delta}}} \tilde{E}_{r}^{( \pm)}(k ; \gamma)
$$

are independent of $r= \pm 1, \pm 3$ and are equal to

$\tilde{E}^{( \pm)}(k)=-2 \pi i \lambda \delta_{ \pm}\left(k^{2}\right)-2 \pi i \frac{\lambda^{2}}{4} \Theta\left( \pm k_{0}\right) \Theta\left(k^{2}\right) \sum_{0}^{\infty} \frac{\left(\frac{\lambda}{4} k^{2}\right)^{m}}{m !(m+1) !(m+2) !}$

$i E_{F, r}(x ; \gamma)$ and $-i E_{\bar{F}, r}(x ; \gamma)$ are time-ordered functions in the following sense:

$$
\begin{array}{r}
i E_{F, r}(x ; \gamma)=\left\{\begin{array}{lll}
i E_{r}^{(+)}(x ; \gamma) & \text { for } & x^{0}>0 \\
i E_{r}^{(+)}(-x ; \gamma) & \text { for } & x^{0}<0
\end{array}\right. \\
-i E_{\bar{F}, r}(x ; \gamma)=\left\{\begin{array}{lll}
i E_{r}^{(+)}(-x ; \gamma) & \text { for } & x^{0}>0 \\
i E_{r}^{(+)}(x ; \gamma) & \text { for } & x^{0}<0
\end{array}\right.
\end{array}
$$

where for instance the first line of Eq. (48) is to be understood as follows: given a test function $f$ with $\tilde{f} \in \mathfrak{M}_{1 / 3}\left(R^{4}\right)$ and with $\operatorname{supp} f \in\left\{x / x^{0}>0\right\}$; then

$$
\int d x f(x) i E_{F, r}(x ; \gamma)=\int d x f(x) i E_{r}^{(+)}(x ; \gamma) .
$$

For $\gamma$ real and larger than nine the contours $L_{\gamma}$ and $L_{\gamma}^{\prime}$ may be opened and pushed to the left such that $-2<\operatorname{Re} s=S_{0}<-\frac{3}{2}$ whence it follows that for these values of $\gamma E_{F(\bar{F}), r}(x ; \gamma)$ and $E_{r}^{( \pm)}(x ; \gamma)$ are locally $L^{2}$ integrable functions of $x$ for which products as for example

$$
\begin{aligned}
& \prod_{1 \leqq j<k \leqq 3}\left[i E_{F, r}\left(x_{j}-x_{k} ; \gamma\right], \prod_{1 \leqq j<k \leqq 3}\left[-i E_{\bar{F}, r}\left(x_{j}-x_{k} ; \gamma\right],\right.\right. \\
& i E_{F, r}\left(x_{i}-x_{j} ; \gamma\right) i E_{r}^{(+)}\left(x_{j}-x_{k} ; \gamma\right) i E_{r}^{(+)}\left(x_{l}-x_{k} ; \gamma\right) \\
& \text { and }-i E_{\bar{F}, r}\left(x_{i}-x_{j} ; \gamma\right) i E_{r}^{(+)}\left(x_{k}-x_{j} ; \gamma\right) i E_{r}^{(+)}\left(x_{k}-x_{i} ; \gamma\right)
\end{aligned}
$$

are unambiguously defined. Formal manipulations which are correct for locally $L^{2}$ integrable functions yield the following relations: 
For $\tilde{f} \in \mathfrak{M}_{1 / 3}\left(R^{12}\right)$

$$
\begin{aligned}
\int \cdots \int \prod_{n=1}^{3} d^{4} x_{n} f\left(x_{1}, x_{2}, x_{3}\right) \\
\quad \cdot\left\{\prod_{1 \leqq j<k \leqq 3}\left[i E_{F, r}\left(x_{j}-x_{k} ; \gamma\right)\right]-\prod_{1 \leqq j<k \leqq 3}\left[-i E_{\bar{F}, r}\left(x_{j}-x_{k} ; \gamma\right)\right]\right\} \\
=\frac{1}{2} \sum_{\sigma \in \mathbb{E}_{3}} \int \cdots \int \prod_{n=1}^{3} d^{4} x_{n} f\left(x_{1}, x_{2}, x_{3}\right) \\
\cdot\left\{\left[i E_{F, r}\left(x_{i}-x_{j} ; \gamma\right)\right]\left[i E_{r}^{(+)}\left(x_{j}-x_{k} ; \gamma\right)\right]\left[i E_{r}^{(+)}\left(x_{i}-x_{k} ; \gamma\right)\right]\right. \\
-\left[-i E_{\bar{F}, r}\left(x_{i}-x_{j} ; \gamma\right]\left[i E_{r}^{(+)}\left(x_{k}-x_{j} ; \gamma\right)\right]\left[i E_{r}^{(+)}\left(x_{k}-x_{i} ; \gamma\right]\right\} .\right.
\end{aligned}
$$

For $\tilde{f} \in \mathfrak{M}_{1 / 3}\left(R^{12}\right)$ with $x_{i}^{0}-x_{k}^{0}>0$ and $x_{j}^{0}-x_{k}^{0}>0$ for all $x_{i}, x_{j}, x_{k} \in \operatorname{supp} f$

$$
\begin{aligned}
& \int \cdots \int \prod_{n=1}^{3} d^{4} x_{n} f\left(x_{1}, x_{2}, x_{3}\right) \prod_{1 \leqq j<k \leqq 3}\left[i E_{F, r}\left(x_{j}-x_{k} ; \gamma\right)\right] \\
&= \int \cdots \int \prod_{n=1}^{3} d^{4} x_{n} f\left(x_{1}, x_{2}, x_{3}\right)\left[i E_{F, r}\left(x_{i}-x_{j} ; \gamma\right)\right]\left[i E_{r}^{(+)}\left(x_{j}-x_{k} ; \gamma\right)\right] \\
& \cdot\left[i E_{r}^{(+)}\left(x_{i}-x_{k} ; \gamma\right)\right] \\
& \int \cdots \int \prod_{n=1}^{3} d^{4} x_{n} f\left(x_{1}, x_{2}, x_{3}\right) \prod_{1 \leqq j<k \leqq 3}\left[-i E_{\bar{F}, r}\left(x_{j}-x_{k} ; \gamma\right)\right] \\
&=\int \cdots \int \prod_{n=1}^{3} d^{4} x_{n} f\left(x_{1}, x_{2}, x_{3}\right)\left[-i E_{\bar{F}, r}\left(x_{i}-x_{j} ; \gamma\right)\right]\left[i E_{r}^{(+)}\left(x_{k}-x_{j} ; \gamma\right)\right] \\
& \quad \cdot\left[i E_{r}^{(+)}\left(x_{k}-x_{i} ; \gamma\right)\right] .
\end{aligned}
$$

In order to establish the connection between

$$
\begin{aligned}
& \prod_{1 \leqq j<k \leqq 3}\left[i E_{F, r}\left(x_{j}-x_{k} ; \gamma\right)\right] \quad \text { and } \quad \mathscr{T}_{r}\left(x_{1}, x_{2}, x_{3} ; \gamma\right) \\
& \prod_{1 \leqq j<k \leqq 3}\left[-i E_{\bar{F}, r}\left(x_{j}-x_{k} ; \gamma\right)\right] \quad \text { and } \quad \overline{\mathscr{T}}_{r}\left(x_{1}, x_{2}, x_{3} ; \gamma\right)
\end{aligned}
$$

for $\gamma$ real and larger than nine we introduce with Speer [4] the functions $E_{F, r, \varepsilon}(x ; \gamma)$ and $E_{\widetilde{F}, r, \varepsilon}(x ; \gamma)$ given by

$$
E_{F(\bar{F}), r, \varepsilon}(k ; \gamma)=\frac{\lambda^{2}}{4} \frac{1}{2 \pi i} \int_{L_{\gamma}} d s \frac{\Gamma(-\gamma(1+s)) \Gamma(-s)}{\Gamma(3+s)} e^{i \pi r s}\left[\frac{\lambda}{4}\left(-k_{(\overline{(})}^{2}, i \varepsilon\right)\right]^{s}, \varepsilon>0 .
$$


Then, the following relations hold in $L_{\mathrm{loc}}^{2}$ and $L_{\mathrm{loc}}^{1}$ respectively:

$$
\lim _{\varepsilon \downarrow 0} E_{F(\bar{F}), r, \varepsilon}(x ; \gamma)=E_{F(\bar{F}), r}(x ; \gamma)
$$

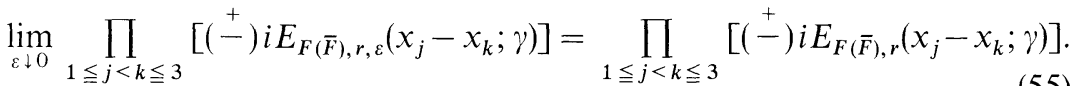

According to Speer we obtain

$$
\begin{aligned}
\mathscr{F}_{x_{1}, x_{2}, x_{3}} & \left\{\prod_{1 \leqq j<k \leqq 3}\left[(\stackrel{+}{-}) i E_{F(\bar{F}), r, \varepsilon}\left(x_{j}-x_{k} ; \gamma\right)\right]\right\}\left(p_{1}, p_{2}, p_{3}\right)=\frac{\pi^{2} \lambda^{4}}{4} \frac{1}{(2 \pi i)^{3}} \\
& \cdot \int_{S_{0}-i \infty}^{S_{0}+i \infty} d s_{3} d s_{2} d s_{1} \prod_{n=1}^{3}\left[\frac{\Gamma\left(-\gamma\left(1+s_{n}\right)\right)}{\Gamma\left(3+s_{n}\right)}\right] \\
& \cdot\left[\frac{\lambda}{4} e^{i \pi r}\right]^{s_{n}+2} \Gamma\left(-\sum_{1}^{3} s_{n}-2\right) \sum_{\sigma \in \Xi_{3}} \delta\left(\sum_{1}^{3} p_{n}\right) \\
& \cdot \int_{0}^{1} d t_{2} t_{2}^{s_{3}+1} \int_{0}^{1} d t_{1} t_{1}^{-s_{1}-1}\left[1+t_{2}+t_{2} t_{1}\right]^{-2} \\
& \cdot\left[\frac{-p_{i}^{2}-t_{1} p_{j}^{2}-t_{2} t_{1} p_{k}^{2}}{1+t_{2}+t_{2} t_{1}}(\mp) i \varepsilon\left(t_{2}^{-1}+1+t_{1}\right)\right]^{\Sigma s_{n}+2}
\end{aligned}
$$

As a consequence of the fact that $-4<\sum_{1}^{3} \operatorname{Re} s_{n}+2<0, \operatorname{Re} s_{3}+1>-1$, uniform convergence of the s-integration and Eq. (16), the limit $\varepsilon \downarrow 0$ of the r.h.s. exists (bounded convergence) and is equal to

$$
\begin{aligned}
& \frac{\pi^{2} \lambda^{4}}{4} \frac{1}{(2 \pi i)^{3}} \int_{S_{0}-i \int_{-\infty}}^{S_{0}+i \infty} d s_{3} d s_{2} d s_{1} \prod_{j=1}^{3}\left[\frac{\Gamma\left(-\gamma\left(1+s_{j}\right)\right)}{\Gamma\left(3+s_{j}\right)}\right] \\
& \cdot\left[\frac{\lambda}{4} e^{i \pi r}\right]^{S_{s_{n}}+2} \Gamma\left(-\sum_{1}^{3} s_{n}-2\right) \sum_{\sigma \in \mathfrak{G}_{3}} \delta\left(\sum_{1}^{3} p_{n}\right) \int_{0}^{1} d t_{2} t_{2}^{s_{3}+1} \int_{0}^{1} d t_{1} t_{1}^{-s_{1}-1} \\
& \cdot\left[1+t_{2}+t_{2} t_{1}\right]^{-2}\left[Q\left(p_{i}, p_{j} ; t_{1}, t_{2}\right)(\overline{+}) i 0\right]^{\Sigma_{n}+2}
\end{aligned}
$$

The limit $\varepsilon \downarrow 0$ of the 1.h.s. of Eq. (56) is given by (55). Thus we find

$$
\prod_{1 \leqq j<k \leqq 3}\left[(\stackrel{+}{-}) i E_{F(\bar{F}), r}\left(x_{j}-x_{k} ; \gamma\right)\right]=\stackrel{(-)}{\mathscr{T}}_{r}\left(x_{1}, x_{2}, x_{3} ; \gamma\right) .
$$

Eq. (58) implies that the left-hand sides of the Eqs. (50), (51) and (51') are analytic functions of $\gamma$ in $W_{\delta}$ and that their limits exist as $\gamma$ tends to +1 from $W_{\delta}$. Also, the products of the right-hand sides of (50), (51) and (51') viewed as convolution integrals in momentum space are analytic functions of $\gamma$ in $W_{\partial}$. This follows since the integrands are analytic in $\gamma$ for $\gamma \in W_{\delta}$ and the integrations are uniformly convergent for $\tilde{f} \in \mathfrak{M}_{1 / 3}\left(R^{12}\right)$ (for $\tilde{f} \in \mathscr{D}\left(R^{12}\right)$ the regions of integration are even compact). The limits of the r.h.s. exist as $\gamma$ tends to +1 from $W_{\partial}$. By the uniqueness of analytic 
continuation in simply connected regions we obtain the desired unitarity and locality relations

$$
\begin{aligned}
& 2 i \int \cdots \prod_{n=1}^{3} d^{4} x_{n} f\left(x_{1}, x_{2}, x_{3}\right) \operatorname{Im} \mathscr{T}\left(x_{1}, x_{2}, x_{3}\right)=\left(\frac{5}{8} \sum_{r= \pm 1}-\frac{1}{8} \sum_{r= \pm 3}\right) \frac{1}{2} \sum_{\sigma \in \Theta_{3}} \\
& \cdot \int \cdots \int \prod_{1}^{3} d^{4} x_{n} f\left(x_{1}, x_{2}, x_{3}\right)\left\{\left[i E_{F, r}\left(x_{i}-x_{j}\right)\right]\left[i E_{r}^{(+)}\left(x_{j}-x_{k}\right)\right]\right. \\
&\left.\cdot\left[i E_{r}^{(+)}\left(x_{i}-x_{k}\right)\right]-\left[-i E_{\bar{F}, r}\left(x_{i}-x_{j}\right)\right]\left[i E_{r}^{(+)}\left(x_{k}-x_{j}\right)\right]\left[i E_{r}^{(+)}\left(x_{k}-x_{i}\right)\right]\right\} \\
&=\frac{1}{2} \sum_{\sigma \in \Theta_{3}} \int \cdots \int \prod_{1}^{3} d^{4} x_{n} f\left(x_{1}, x_{2}, x_{3}\right)\left\{\left[i E_{F}\left(x_{i}-x_{j}\right)\right]\left[i E^{(+)}\left(x_{j}-x_{k}\right)\right]\right. \\
&\left.\cdot\left[i E^{(+)}\left(x_{i}-x_{k}\right)\right]-\left[-i E_{\bar{F}}\left(x_{i}-x_{j}\right)\right]\left[i E^{(+)}\left(x_{k}-x_{j}\right)\right]\left[i E^{(+)}\left(x_{k}-x_{i}\right)\right]\right\}
\end{aligned}
$$

etc. Here the Eqs. (33), (41), (44) and (44') have been used. This completes the proof that the above definitions of $\mathscr{T}$ and $\overline{\mathscr{T}}$ are permissible.

Finally, we want to point out a special property of these definitions. For $p_{l}^{2}>0, l=1,2,3$, the real part of $\tilde{\mathscr{T}}\left(p_{1}, p_{2}, p_{3}\right)$ can be brought into the form

$$
\begin{aligned}
& \frac{\pi^{2} \lambda^{4}}{4} \frac{1}{(2 \pi i)^{3}} \int_{S-i \infty}^{S+i \infty} d s_{3} d s_{2} d s_{1} \prod_{n=1}^{3}\left[\frac{\Gamma\left(-1-s_{n}\right)}{\Gamma\left(3+s_{n}\right)}\right]\left(\frac{\lambda}{4}\right)^{\Sigma s_{n}+2} \Gamma\left(-\sum_{1}^{3} s_{n}-2\right) \\
& \cdot \sum_{\sigma \in \Theta_{3}} \delta\left(\sum_{1}^{3} p_{n}\right) \int_{0}^{1} d t_{2} t_{2}^{s_{3}+1} \int_{0}^{1} d t_{1} t_{1}^{-s_{1}-1}\left[1+t_{2}+t_{2} t_{1}\right]^{-\Sigma s_{n}-4} \\
& \cdot\left[p_{i}^{2}+t_{1} p_{j}^{2}+t_{1} t_{2} p_{k}^{2}\right]^{s_{n}+2}
\end{aligned}
$$

or into the form

$$
\begin{aligned}
& \frac{\pi^{2} \lambda^{4}}{4} \frac{1}{2 \pi i} \int_{T-i \infty}^{T+i \infty} d s\left(\frac{\lambda}{4}\right)^{s} \Gamma(-s) \frac{1}{(2 \pi i)^{2}} \int_{U-i \infty}^{U+i \infty} d s_{3} d s_{1} \prod_{j=1,3}\left[\frac{\Gamma\left(-1-s_{j}\right)}{\Gamma\left(3+s_{j}\right)}\right] \\
& \cdot \frac{\Gamma\left(s_{3}+s_{1}+1-s\right)}{\Gamma\left(1+s-s_{1}-s_{3}\right)} \sum_{\sigma \in \mathfrak{S}_{3}} \delta\left(\sum_{1}^{3} p_{n}\right) \int_{0}^{1} d t_{2} t_{2}^{s_{3}+1} \int_{0}^{1} d t_{1} t_{1}^{-s_{1}-1} \\
& \cdot\left[1+t_{2}+t_{2} t_{1}\right]^{-s-2}\left[p_{i}^{2}+t_{1} p_{j}^{2}+t_{1} t_{2} p_{k}^{2}\right]^{s}
\end{aligned}
$$

where $U=-\frac{3}{2}, T>-3$.

Simple estimates using Stirling's formula show that $\operatorname{Re} \tilde{\mathscr{T}}$ tends to zero in the region $\mathcal{O}$

$$
\mathcal{O}=\left\{p_{1}, p_{2}, p_{3} / p_{j}^{2}>0 \quad j=1,2,3\right\}
$$

as the invariant momenta $p_{l}^{2}$ tend to $+\infty$ with a decrease of type

$$
\delta\left(\sum_{1}^{3} p_{n}\right) \cdot O\left(\operatorname{Max}_{l=1,2,3}^{-5 / 2} p_{l}^{2}\right)
$$


and

$$
\delta\left(\sum_{1}^{3} p_{n}\right) \cdot O\left(\operatorname{Min}_{l=1,2,3}^{-3} p_{l}^{2}\right) .
$$

This fall-off property fixes the time-ordered function completely. For, any other choice would differ from our definition in momentum space by a real entire function of $p_{1}^{2}, p_{2}^{2}, p_{3}^{2}$ of order $<\frac{1}{2}$. These entire functions do not decrease in any direction in $\mathbb{C}^{3}$. Thus the real part of any other choice of the time-ordered function does not decrease in momentum space in any direction inside the region $\mathcal{O}$.

\section{Naturalness of the Definition}

In this section we want to illustrate that the choice (31) of the product of the three superpropagators is the most natural one.

We note that the imaginary part of this product is completely fixed by unitarity. Its real part is fixed by locality away from the point where all coordinates coincide, the difference between two admissible choices being a real distribution concentrated in that point. We confine our attention to the real part of the product since it is this part that contains all the ambiguities and needs precise definition.

We consider the function $\tau^{f}\left(t_{1}, t_{2}, t_{3}\right)$ of the time differences $t_{j}-t_{k}$ that arises from $\operatorname{Re} \mathscr{T}\left(x_{1}, x_{2}, x_{3}\right)$, given by (31), after smearing over the spatial variables with test functions $f: \tilde{f} \in \mathfrak{M}_{1 / 3}\left(R^{9}\right)$. In order to determine the detailed structure of this function we need more information about $\operatorname{Re} \tilde{\mathscr{T}}$ than the previously established decrease $O\left(\operatorname{Min}_{j=1,2,3}^{-3} p_{j}^{2}\right)$ and $O\left(\operatorname{Max}_{j=1,2,3}^{-5 / 2} p_{j}^{2}\right)$ in $\mathcal{O}=\left\{p_{1}, p_{2}, p_{3} / p_{j}^{2}>0 j=1,2,3\right\}$. We have to know the behavior of $\operatorname{Re} \tilde{\mathscr{T}}$ in $\mathscr{A}_{K^{2}}^{l}$

$\mathscr{A}_{K^{2}}^{l}=\left\{p_{1}, p_{2}, p_{3} /-K^{2}<p_{l}^{2}<0, p_{m}^{2}>0, p_{n}^{2}>0\right\}, \quad 0<K^{2}<\infty$

as $p_{m}^{2}$ (and $p_{n}^{2}$ ) tend to $+\infty$. To this end, we study the following function

$$
\begin{aligned}
& F\left(x_{1}, x_{2}, x_{3}\right)=\sum_{0}^{\infty} \frac{1}{m_{3} !\left(m_{3}+1\right) !} \sum_{0}^{\infty} \frac{1}{m_{2} !\left(m_{2}+1\right) !} \frac{1}{2 \pi i} \int_{C} d s_{1} \frac{\Gamma\left(-1-s_{1}\right)}{\Gamma\left(3+s_{1}\right)} \\
& \cdot \cos \pi s_{1}\left[1+\sin ^{2} \pi s_{1}\right] \Gamma\left(-m_{3}-m_{2}-s_{1}\right) \Gamma\left(-s_{1}\right) \\
& \quad \text { analyt. cont. } \Gamma\left(-s_{1}\right)^{-1} \int_{0}^{1} d t_{2} t_{2}^{m_{3}} \int_{0}^{1} d t_{1} t_{1}^{-s_{1}-1}\left[1+t_{2}+t_{2} t_{1}\right]^{-m_{3}-m_{2}-s_{1}-2} \\
& \quad \cdot \frac{1}{2}\left\{\left[x_{1}-t_{1}\left(x_{2}+t_{2} x_{3}\right)-i 0\right]^{m_{3}+m_{2}+s_{1}}+\left[x_{1}-t_{1}\left(x_{2}+t_{2} x_{3}\right)+i 0\right]^{m_{3}+m_{2}+s_{1}}\right\} \\
& +\int_{0}^{1} d t_{2} \int_{0}^{1} d t_{1} \mathfrak{R}\left(x_{1}, x_{2}, x_{3} ; t_{1}, t_{2}\right)
\end{aligned}
$$


where

$$
\begin{gathered}
\Re\left(x_{1}, x_{2}, x_{3} ; t_{1}, t_{2}\right)=\sum_{l=0}^{\infty} \frac{\left[x_{1}-t_{1}\left(x_{2}+t_{2} x_{3}\right)\right]^{l}}{l !\left[1+t_{2}+t_{2} t_{1}\right]^{l+2}} \frac{1}{(2 \pi i)^{2}} \int_{S_{2}-i \infty}^{s_{2}+i \infty} d s_{2} \\
\quad \int_{S_{1}-i \infty}^{s_{1}+i \infty} d s_{1} \prod_{j=1}^{2}\left[\frac{\Gamma\left(-1-s_{j}\right)}{\Gamma\left(3+s_{j}\right)}\right] \frac{\Gamma\left(s_{2}+s_{1}+1-l\right)}{\Gamma\left(1+l-s_{1}-s_{2}\right)} t_{2}^{l-1-s_{1}-s_{2}} t_{1}^{-s_{1}-1}, \\
S_{2}<-1, S_{1}<-2,-7 / 2<S_{2}+S_{1}<-3 .
\end{gathered}
$$

We are interested in the behavior of $F\left(x_{1}, x_{2}, x_{3}\right)$ for $x_{j}>0 j=1,2,3, x_{2}$ (and $x_{3}$ ) tending to $+\infty$ and $x_{1}$ staying finite $0<x_{1}<K^{2}$. We split the $t_{1}$ integrations into three parts: 1) from 0 to $\left.\xi\left(t_{2}\right), 2\right)$ from $\xi\left(t_{2}\right)$ to $2 \xi\left(t_{2}\right)$ and 3) from $2 \xi\left(t_{2}\right)$ to +1 where $\xi\left(t_{2}\right)=\frac{x_{1}}{x_{2}+t_{2} x_{3}}$. Accordingly, we write $F\left(x_{1}, x_{2}, x_{3}\right)$ as a sum of three terms $F_{j}\left(x_{1}, x_{2}, x_{3}\right)$ corresponding to the respective intervals. It is not difficult to determine the behavior of the terms $F_{3}$ and $F_{2}$ which can be cast into the form

$$
\begin{aligned}
& F_{3}\left(x_{1}, x_{2}, x_{3}\right)=\int_{0}^{1} d t_{2} \int_{0}^{\xi\left(t_{2}\right)} \int_{1}^{-1}-2 \frac{1}{(2 \pi i)^{3}} \int_{s-i \infty}^{s+i \infty} \int_{-\infty}^{\infty} d s_{3} d s_{2} d s_{1} \prod_{1}^{3}\left[\frac{\Gamma\left(-1-s_{j}\right)}{\Gamma\left(3+s_{j}\right)}\right] \\
& \cdot \Gamma\left(-\sum_{1}^{3} s_{j}-2\right) t_{2}^{s_{3}+1}\left(2+t_{1}\right)^{-s_{1}-1}\left(1+t_{1}\right)^{s_{j}+2} \\
& \cdot\left[1+t_{2}+t_{2}\left(2+t_{1}\right) \cdot \xi\left(t_{2}\right)\right]^{-s_{j}-4} x_{1}^{s_{3}+s_{2}+2}\left[x_{2}+t_{2} x_{3}\right]^{s_{1}} \\
& F_{2}\left(x_{1}, x_{2}, x_{3}\right)=\int_{0}^{1} d t_{2} \sum_{0}^{\infty} \frac{\left(-x_{1} t_{2}\right)^{m_{3}}}{m_{3} !\left(m_{3}+1\right) !} \sum_{0}^{\infty} \frac{\left(-x_{1}\right)^{m_{2}}}{m_{2} !\left(m_{2}+1\right) !} \\
& \frac{1}{2 \pi i} \int_{S_{1}-i \infty}^{s_{1}+i \infty} d s_{1} \frac{\Gamma\left(-1-s_{1}\right)}{\Gamma\left(3+s_{1}\right)} \frac{\pi^{s}}{\sin \pi s_{1}} \Gamma\left(-m_{3}-m_{2}-s_{1}\right)\left[x_{2}+t_{2} x_{3}\right]^{s_{1}} \\
& \cdot \frac{1}{2 \pi i} \int_{1}^{0+} d t_{1}\left(-t_{1}\right)^{m_{3}+m_{2}+s_{1}}\left[1+t_{1}\right]^{-s_{1}-1}\left[1+t_{2}+t_{2}\left(1+t_{1}\right) \cdot \xi\left(t_{2}\right)\right]^{-m_{3}-m_{2}-s_{1}-2} \\
& +\int_{0}^{1} d t_{2} \int_{\xi\left(t_{2}\right)} d t_{1} \mathfrak{R}\left(x_{1}, x_{2}, x_{3} ; t_{1}, t_{2}\right) .
\end{aligned}
$$

Clearly, $F_{3}$ is of type $O\left(x_{2}^{-5 / 2}\right)$. It is an immediate consequence of the definition of $\mathfrak{R}\left(x_{1}, \ldots, t_{2}\right)$ that the second term of the r.h.s. of $(67)$ is of 
type $O\left(x_{2}^{-5 / 2}\right)$. Hence, also $F_{2}$ is of type $O\left(x_{2}^{-5 / 2}\right) . F_{1}$ is given by

$$
\begin{aligned}
& F_{1}\left(x_{1}, x_{2}, x_{3}\right)=\int_{0}^{1} d t_{2} \sum_{0}^{\infty} \frac{\left(x_{1} t_{2}\right)^{m_{3}}}{m_{3} !\left(m_{3}+1\right) !} \sum_{0}^{\infty} \frac{x_{1}^{m_{2}}}{m_{2} !\left(m_{2}+1\right) !} \frac{1}{2 \pi i} \\
& \cdot \int_{C} d s_{1} \frac{\Gamma\left(-1-s_{1}\right)}{\Gamma\left(3+s_{1}\right)} \frac{\pi \cos \pi s_{1}}{\sin \pi s_{1}}\left[1+\sin ^{2} \pi s_{1}\right] \Gamma\left(-m_{3}-m_{2}-s_{1}\right)\left[x_{2}+t_{2} x_{3}\right]^{s_{1}} \\
& \cdot\left\{\frac{1}{2 \pi i} \int_{Z_{r}} d t_{1}\left(-t_{1}\right)^{-s_{1}-1}\left(1-t_{1}\right)^{m_{3}+m_{2}+s_{1}}\left[1+t_{2}+t_{2} t_{1} \cdot \xi\left(t_{2}\right)\right]^{-m_{3}-m_{2}-s_{1}-2}\right\} \\
& +\int_{0}^{1} d t_{2} \int_{0}^{\xi\left(t_{2}\right)} d t_{1} \mathfrak{R}\left(x_{1}, x_{2}, x_{3} ; t_{1}, t_{2}\right)
\end{aligned}
$$

where $Z_{r}$ is the circle of radius $r$ around the origin with positive orientation, $r$ larger than one and fixed.

Again, the second term of the r.h.s. is of type $O\left(x_{2}^{-5 / 2}\right)$. We note that the curled bracket is bounded by $2^{m_{3}+m_{2}+\operatorname{Re} s_{1}} \cdot \exp \left[\frac{\pi}{3}\left|\operatorname{Im} s_{1}\right|\right]$. The first term of the r.h.s. of (68) may be written as the sum of two terms according to the factor $\left[1+\sin ^{2} \pi s_{1}\right]$. In the term corresponding to 1 the contour $C$ may be straightened out which leads to a behavior of type $O\left(x_{2}^{-5 / 2}\right)$ for this term. In the term corresponding to $\sin ^{2} \pi s_{1}$ the $s_{1}$ and $t_{1}$ integrations may be replaced by summations over the respective residues which leads to an entire function of $x_{2}$ and $x_{3}$ of order 1/3. Summarizing, the behavior of $F\left(x_{1}, x_{2}, x_{3}\right)$ for $x_{j}>0 j=1,2,3$ as $x_{2}$ (and $x_{3}$ ) tends to $+\infty$ and $x_{1}$ stays finite: $0<x_{1}<K^{2}$ is given by a sum of an entire function of $x_{2}$ and $x_{3}$ of order $1 / 3$ plus a function of type $O\left(x_{2}^{-5 / 2}\right)$.

Now we are in a position to determine the behavior of $\operatorname{Re} \tilde{\mathscr{T}}\left(p_{1}, p_{2}, p_{3}\right)$ when some of the time components of the momenta tend to $\pm \infty$ while the space components are fixed in a compact set. Due to the presence of the energy conservation $\delta$-function there are only two alternatives

1) $p_{j, 0}^{2} \rightarrow+\infty$ for $j=1,2,3$.

2) $p_{l, 0}^{2}$ stays finite, $p_{m, 0}^{2}$ and $p_{n, 0}^{2}$ tend to $+\infty$.

In the first case all $p_{j}^{2}$ tend to $+\infty$ which is a situation we have already dealt with in the preceding section where we found that $\operatorname{Re} \tilde{\mathscr{T}}\left(p_{1}, p_{2}, p_{3}\right)=\delta\left(\sum_{1}^{3} p_{n}\right) O\left(\operatorname{Min}_{j=1,2,3}^{-3} p_{j}^{2}\right)$. In the second case $p_{m}^{2}$ and $p_{n}^{2}$ tend to $+\infty$ while $p_{l}^{2}$ stays finite. If $p_{l}^{2}>0$ we invoke once again a result of the preceding section and obtain

$$
\operatorname{Re} \tilde{\mathscr{T}}\left(p_{1}, p_{2}, p_{3}\right)=\delta\left(\sum_{1}^{3} p_{n}\right) O\left(\operatorname{Max}^{-5 / 2}\left\{p_{m}^{2}, p_{n}^{2}\right\}\right)
$$


If $p_{l}^{2}<0$ we go back to the definition of $\tilde{\mathscr{T}}$, formula (31), and split the sum $\sum_{\sigma \in \mathfrak{G}_{3}}$ into two terms according to whether $i \neq l$ or $i=l$. The term with $i \neq l$ can be brought into a form analogous to (60) leading again to a behavior of type $\delta\left(\sum_{1}^{3} p_{n}\right) \cdot O\left(\operatorname{Max}^{-5 / 2}\left\{p_{m}^{2}, p_{n}^{2}\right\}\right)$ whereas the term corresponding to $i=l$ can be expressed by means of $F\left(x_{1}, x_{2}, x_{3}\right)$ and is equal to

$$
\delta\left(\sum_{1}^{3} p_{n}\right) \frac{\pi^{2} \lambda^{4}}{4}\left\{F\left(-\frac{\lambda}{4} p_{l}^{2}, \frac{\lambda}{4} p_{m}^{2}, \frac{\lambda}{4} p_{n}^{2}\right)+F\left(-\frac{\lambda}{4} p_{l}^{2}, \frac{\lambda}{4} p_{n}^{2}, \frac{\lambda}{4} p_{m}^{2}\right)\right\} .
$$

The behavior of $F\left(x_{1}, x_{2}, x_{3}\right)$ in the region of interest has been established above. Thus we see that the behavior of $\operatorname{Re} \tilde{\mathscr{T}}\left(p_{1}, p_{2}, p_{3}\right)$ for $p_{l}^{2}<0$, $p_{m}^{2}$ and $p_{n}^{2}$ tending to $+\infty$ is given by

$$
\delta\left(\sum_{1}^{3} p_{r}\right)\left\{g\left(p_{l}^{2}, p_{m}^{2}, p_{n}^{2}\right)+O\left(\left(p_{m}^{2}\right)^{-5 / 2}\right)+O\left(\left(p_{n}^{2}\right)^{-5 / 2}\right)\right\}
$$

where $g$ is an entire function of $p_{m}^{2}$ and $p_{n}^{2}$ of order $1 / 3$.

Combining the various pieces of information, we obtain for $\tilde{f} \in \mathscr{D}\left(R^{9}\right)$

$$
\begin{aligned}
\hat{\tau}^{f}\left(p_{1.0}, p_{2,0}, p_{3,0}\right)=\int \cdots \int \prod_{1}^{3} d^{3} \boldsymbol{p}_{j} \tilde{f}\left(\boldsymbol{p}_{1}, \boldsymbol{p}_{2}, \boldsymbol{p}_{3}\right) \operatorname{Re} \tilde{\mathscr{T}}\left(p_{1}, p_{2}, p_{3}\right) \\
=\delta\left(\sum_{1}^{3} p_{n, 0}\right)\left\{O\left(\operatorname{Min}\left\{\operatorname{Min}_{j}^{-6}\left|p_{j, 0}\right|, \operatorname{Max}_{j}{ }^{-5}\left|p_{j, 0}\right|\right\}\right)+\sum_{\sigma \in \mathbb{E}_{3}} \Theta\left(E-\left|p_{i, 0}\right|\right) \cdot\right. \\
\left.\quad \cdot\left[O\left(\left|p_{j, 0}\right|^{-5}\right)+O\left(\left|p_{k, 0}\right|^{-5}\right)+\hat{g}\left(p_{i, 0}, p_{j, 0}, p_{k, 0}\right)\right]\right\} .
\end{aligned}
$$

Here $E$ is some constant depending on the support of $\tilde{f}$ and $\hat{g}\left(p_{i, 0}, p_{j, 0}, p_{k, 0}\right)$ is an entire function of $p_{j, 0}$ and $p_{k, 0}$.

The statement (69) implies the following structure of $\tau^{f}\left(t_{1}, t_{2}, t_{3}\right)$ :

$$
\tau^{f}\left(t_{1}, t_{2}, t_{3}\right)=h^{f}\left(t_{1}-t_{2}, t_{2}-t_{3}, t_{3}-t_{1}\right)+\sum_{\sigma \in \mathbb{E}_{3}} \sum_{v=0}^{\infty} \delta^{(v)}\left(t_{i}-t_{j}\right) h_{v, \sigma}^{f}\left(t_{j}-t_{k}\right)
$$

for $\tilde{f} \in \mathscr{D}\left(R^{9}\right)$ where $h^{f}$ is a three times continuously differentiable function and $h_{v, \sigma}^{f}$ are infinitely differentiable functions such that

$$
\sum_{v=0}^{\infty} \delta^{(v)}\left(t_{i}-t_{j}\right) h_{v, \sigma}^{f}\left(t_{j}-t_{k}\right) \in \mathscr{F} \mathfrak{M}_{1,3}^{\prime}\left(R^{2}\right) .
$$

This result does not only hold for $\tilde{f} \in \mathscr{D}\left(R^{9}\right)$. Due to uniform convergence of the $\boldsymbol{p}_{i}$-integrations it also holds $\tilde{f} \in \mathfrak{M}_{1 / 3}\left(R^{9}\right)$.

The singularities of $\tau^{f}$, dictated by locality, are contained in the second term of the r.h.s. of (70). They are attached to the lines $t_{1}=t_{2}$, 
$t_{2}=t_{3}$ and $t_{3}=t_{1}$. For no $\tilde{f} \in \mathfrak{M}_{1 / 3}\left(R^{9}\right)$ does $\tau^{f}$ possess any singularity attached exclusively to the point $t_{1}=t_{2}=t_{3}$. Any other choice of the time-ordered function would lead to a corresponding $\tau^{f}$ possessing such a point singularity for at least one $f$ with $\tilde{f} \in \mathfrak{M}_{1 / 3}\left(R^{9}\right)$.

\section{Conclusions}

The crucial property of a certain class of non-polynomial Lagrangians to which the exponential Lagrangian belongs is that in the weighted space averages of the time-ordered two- and three-point functions background (line singularities) and point singularity can be separated from each other. The special feature of our particular choices of the time-ordered two- and three-point functions consist in the absence of point singularities or in other words in their being least singular. However, it should be mentioned that the triangle graph considered here is a relatively simple object and enjoys special properties that need not be true for the general perturbation theoretic term.

Finally, it is worth noting that in spite of the extremely singular nature of the exponential interaction the analyticity structure of the triangle graph in the invariant momenta on all sheets of the Riemann surface is just the same as in renormalizable models. This is so because $\tilde{\mathfrak{R}}^{-}$is an entire function and the sums and the integral in the first term of the r.h.s. of (31) converge uniformly in a neighborhood of any point of analyticity of $T_{0}^{\sigma-}\left(\{p\} ; s_{1}, m_{2}-1, m_{3}-1\right) / \Sigma p_{j}=0$ regarded as a funtion on the complex invariant momenta. Thus the singularities are determined by $T_{0}^{\sigma-}\left(\{p\} ; s_{1}, m_{2}-1, m_{3}-1\right) / \sum p_{3}=0$, their location is independent of $s_{1}, m_{2}$ and $m_{3}$ and hence the same as in conventional theories.

Acknowledgement. The author is greatly indebted to Prof. H. Lehmann for his valuable advice and constant stimulating encouragement during the progress of this work. It is a pleasure to thank Dr. Carl Kaysen and the faculty for their hospitality at the Institute for Advanced Study.

\section{References}

1. Lehmann, H., Pohlmeyer, K.: Commun. math. Phys. 20, 101 (1971) and references quoted there.

2. Jaffe, A.: Phys. Rev. 158, 1454 (1967) and unpublished manuscript.

3. Gelfand, I. M., Schilow, G. E.: Verallgemeinerte Funktionen (Distributionen) I. Berlin: VEB Deutscher Verlag der Wissenschaften 1960.

4. Speer,E. R.: J. Math. Phys. 9, 1404 (1968).

K. Pohlmeyer

II. Institut für Theoretische Physik

der Universität

D-2000 Hamburg 50

Luruper Chaussee 149

Germany 